\title{
Dioscin inhibits ischemic stroke-induced inflammation through inhibition of the TLR4/MyD88/NF-кB signaling pathway in a rat model
}

\author{
SHILIN ZHU ${ }^{1}$, SIYUAN TANG ${ }^{2}$ and FENG SU ${ }^{3}$ \\ ${ }^{1}$ Department of Neurology, The Second Affiliated Hospital, Hunan University of Chinese Medicine, \\ Changsha, Hunan 410005; ${ }^{2}$ Xiang Ya Nursing School of Central South University, Changsha, Hunan 410013; \\ ${ }^{3}$ Department of Emergency, Xiang Ya Hospital, Central South University, Changsha, Hunan 410008, P.R. China
}

Received August 28, 2016; Accepted May 23, 2017

DOI: $10.3892 / \mathrm{mmr} .2017 .7900$

\begin{abstract}
Diosgenin, as an essential natural steroidal saponin, can be extracted from numerous sources, primarily from fenugreek. It is an important raw material for the synthesis of steroid hormone drugs. It exhibits antitumor, anti-inflammatory, antioxidation and several other significant pharmacologic actions, and is of high pharmaceutical value. In the present study, the activities and underlying mechanisms of dioscin in the inhibition of ischemic stroke in rats were investigated. Inflammatory responses wer analyzed using ELISA kits and caspase-3 and caspase- 9 activity was analyzed using Caspase- 3 and caspase- 9 activity kits. Western blot analysis was used to measure Toll-like receptor 4 (TLR4), myeloid differentiation factor 88 (MyD88), nuclear factor- $\kappa \mathrm{B}$ (NF- $\kappa \mathrm{B})$, transforming growth factor- $\beta 1$ (TGF- $\beta 1$ ), high-mobility group protein 1 (HMGB-1), interleukin-1 receptor-associated kinase 1 (IRAK1), and tumor necrosis factor receptor-associated factor 6 (TRAF6) protein expression. Dioscin inhibited infarct volume and neurological scores in the ischemic stroke rat model. The results demonstrated that dioscin reduced inflammatory responses, and suppressed the expression of TLR4, MyD88, NF- $\mathrm{kB}$, TGF- $\beta 1$, HMGB-1, IRAK1, and TRAF6 in the rat ischemic stroke model. Taken together, these findings suggested that dioscin inhibited ischemic stroke-induced inflammation through inhibition of the TLR4/MyD88/NF-kB-induced inflammation the rat model, which provided novel insights into the mechanisms
\end{abstract}

Correspondence to: Mr. Siyuan Tang, Xiang Ya Nursing School of Central South University, 172 Tongzipo Road, Yuelu, Changsha, Hunan 410013, P.R. China

E-mail: taozongya0536008@126.com

Dr Feng Su, Department of Emergency, Xiang Ya Hospital, Central South University, 87 Xiang Ya Road, Kaifu, Changsha, Hunan 410008, P.R. China

E-mail: sf251@sina.com

Key words: dioscin, ischemic stroke, inflammation, Toll-like receptor 4 , myeloid differentiation factor 88 , nuclear factor- $\kappa \mathrm{B}$ underlying the effect of dioscin as an anti-inflammatory candidate for the treatment of ischemic stroke in in the future.

\section{Introduction}

Ischemic stroke is the most common type of cerebrovascular disease, accounting for $85 \%$ of all cerebrovascular diseases. It is ranked third among life-threatening diseases and is the most disabling disease worldwide (1). It severely affects human health and, within 3 months following a stroke, 15-30\% of survivors suffer from permanent disability, including paralysis, memory disorders, thought disorders, linguistic problems and akinesia (2). Of these individuals, $\sim 20 \%$ require care as they cannot perform self-care. In western countries, $>70 \%$ of the population $>65$ years old experience a stroke (3), and the number of patients continues to increase (3). Although the pathology and physiology of ischemic stroke involve different mechanisms, increasing evidence has indicated that ischemic damage and inflammation lead to disease progression (4). Cerebral ischemia induces pathological pathways in an ischemia cascade reaction and causes irreversible damage of ischemic core neurons (5).

At present, substantial evidence has shown that the essential mechanism of cerebral ischemic injury involves oxidative stress and inflammatory reaction (6). The use of psychotherapeutic drugs for anti-inflammatory treatment has provided scope clinically, including for the treatment of tumors (7), and it has shown preliminary treatment effects. Consequently, theoretical and clinical investigations aimed at enhancing the inflammatory mechanism following cerebral ischemia is likely to generate novel opportunities for the treatment of cerebral infarction $(6,8)$.

Toll-like receptor 4 (TLR4) is a transmembrane receptor protein composed of an extracellular region, transmembrane region and intracellular region (9). Through recognition of high-mobility group protein 1 (HMGB1), heat shock protein, fibrous protein, necrocytosis components and other molecules released at sites of histocyte damage, TLR4 further activates the signal transduction pathway in the cell to enhance the synthesis and release of cytokines, promoting the maturation and differentiation of immune cells and regulating the immune response (9). In peripheral regions, TLR4 is predominantly distributed on 
lymphocytes, macrophages, dendritic cells and other innate immunocytes, and is involved in immune responses to bacteria and other exogenous pathogens. In the central nervous system, TLR4 distributes extensively on astrocytes, microglial cells, vascular endothelium and smooth muscle cytomembranes, and is involved in the pathogenic process of Parkinson's disease, Alzheimer's disease and other neurodegenerative diseases (10). The activated TLR 4 can activate nuclear factor $-\kappa \mathrm{B}(\mathrm{NF}-\kappa \mathrm{B})$ in a resting state, which translocates to the cell nucleus to initiate the gene expression of TNF-(4 interleukin (IL)-1, cyclooxygenase-2 (COX-2) and other adhesion molecules through combining with myeloid differentiation antigen 88 (MyD88), leading to inflammatory reactions (11).

Diosgenin, generally known as saponin, is a natural and synthetic steroid sapogenin belonging to a screw sterane alcohol glucoside (Fig. 1) (12). The relative molecular mass is 414.63 , and is present extensively in leguminosae and dioscoreaceae plants. Diosgenin is found in the seeds of fenugreek Trigonella foenum-graecum L. (13). It can also be extracted from the tuber of Dioscorea zingiberensis C. H. Wright, D. nipponica Makino, D. panthaica Prain et Burkill and D. nipponica Makino ssp. Rosthornii (Prain et Burkill) C. T. Ting through methods, including hydrolyzation, fermentation and extraction (14). Diosgenin, as an essential raw material for the synthesis of steroid hormone drugs and steroidal contraceptives, is used to produce pregnenolone, progesterone and cortisone (15). Previous in-depth investigations of the pharmacologic actions of diosgenin have been performed. Diosgenin has antitumor effects in addition to blood lipid-regulating, anti-platelet aggregating and bilifaction-promoting effects (14). It is an essential drug for the treatment of cardiovascular disease, encephalitis, dermatosis and tumors $(15,16)$. Therefore, the aim of the present study was to investigate the neuroprotective effect of dioscin on inhibiting the effects of ischemic stroke and its possible mechanisms.

\section{Materials and methods}

Animals. Adult male Sprague-Dawley rats (8-10 weeks old, 200-230 g) were purchased from the Hunan Experimental Animal Centre of Hunan University of Chinese Medicine (Hunan, China) and fed a commercially available liquid diet. The rats were housed in separate cages in a room with controlled temperature $\left(22-24^{\circ} \mathrm{C}\right), 12 \mathrm{~h}$ light:dark cycle (light between 8:00 and 20:00) and humidity (50-55\%). The protocols used in the present study followed the National Institutes of Health guidelines for the Care and Use of Laboratory Animals (National Institutes of Health, Bethesda, MD, USA), were approved by the institutional animal ethics committee of Hunan University of Chinese Medicine of Medicine. Following 1 week of acclimatization, the rats were randomly divided into three groups ( $n=8$ per group) as follows: Sham group, stroke model group, and dioscin treatment group. In the dioscin treatment group, the rats were intragastrically treated with $80 \mathrm{mg} / \mathrm{kg} /$ day of dioscin for 4 weeks.

Middle cerebral artery occlusion (MCAO). All rats were anesthetized with $2.5 \%$ isoflorane (Forane; Abbot Japan, Tokyo, Japan) and were maintained at $37 \pm 1^{\circ} \mathrm{C}$ during the

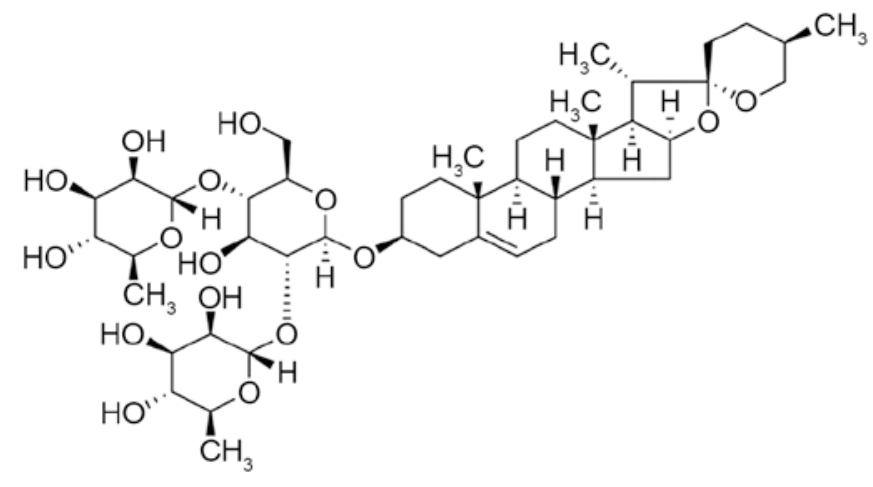

Figure 1. Structural formula of dioscin.

experiment. The right common carotid artery was exposed using a midline neck incision, and 4-cm poly-L-lysine-coated nylon thread (3-0) was inserted into the internal carotid artery through the right common carotid artery and gently advanced until resistance was detected in the blood flow trace. MCAO was maintained for $2 \mathrm{~h}$, following which the thread was gently removed to restore blood flow. The wounds were sterilized and sutured, and the rats were allowed to recover from anesthesia.

Neurological assessment. The modified neurological severity score was used to assess neurologic severity scores. The neurologic function was graded with scores between 0 and 5 ( 0 , no neurologic deficit; 5, maximal deficit). A score of 1 indicated that the rats were unable perform the test or the tested reflex, and a higher score indicated more severe injury.

Following anesthesia with $2.5 \%$ isoflorane, the rats were sacrificed and their brains were removed, cleaned and solidified using pre-cooled normal saline $\left(4^{\circ} \mathrm{C}\right)$ for $5 \mathrm{~min}$. The prepared slices $(5 \mu \mathrm{M})$ were stained with $2 \%$ 2,3,5-triphenyltetrazolium chloride (Sigma; Merck Millipore; Darmstadt, Germany) and fixed in $10 \%$ buffered formalin solution. Images were captured using light microscopy (Nikon Eclipse TE2000-U; Nikon, Tokyo, Japan).

Determination of biological indicators. Following anesthesia with $2.5 \%$ isoflorane, the rats were sacrificed, following which their brains were removed and cleaned, and the hippocampus was separated. Total cellular protein was lysed from the hippocampal tissue using radioimmunoprecipitation lysis buffer (Beyotime Institute of Biotechnology, Jiangsu, China) and the protein content was determined using a BCA protein assay kit (Beyotime Institute of Biotechnology). The proteins (2-5 inate of Biotechnologyionthe activities of IL-1 activities of Biotechusing ELISA kits. The color intensity was measured at $450 \mathrm{~nm}$. The proteins (10 $\mu$ ot were incubated with the caspase-3 and caspase- 9 activity kits (Ac-DEVD-pNA and Ac-LEHD-pNA) for $2 \mathrm{~h}$ at $37^{\circ} \mathrm{C}$. The color intensity was measured at $405 \mathrm{~nm}$.

Western blot analysis. Total cellular protein was lysed from the hippocampal tissue using radioimmunoprecipitation lysis buffer (Beyotime Institute of Biotechnology) and the protein content was determined using a BCA protein assay kit (Beyotime Institute of Biotechnology). The proteins $(50 \mu \mathrm{g})$ were subjected to $8-12 \%$ SDS-PAGE and then transferred 

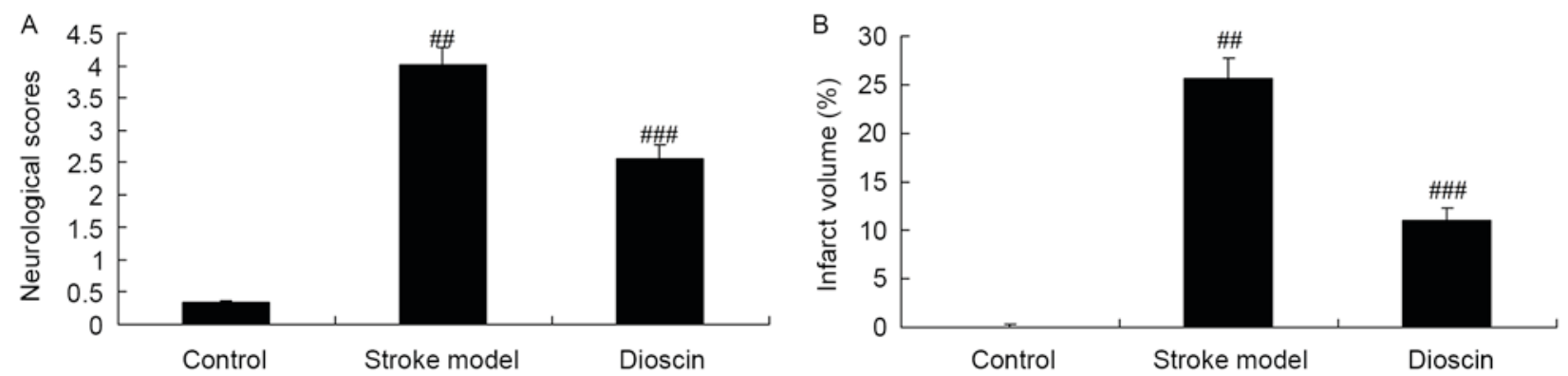

Figure 2. Effect of dioscin on infarct volume and neurological scores in ischemic stroke rats. Effects of dioscin on (A) infarct volume and (B) neurological scores in ischemic stroke rats. Control, control group; Stroke, ischemic stroke model group; Dioscin, dioscin treatment group. ${ }^{\# \#} \mathrm{P}<0.01$, vs. control group;

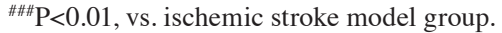

onto a nitrocellulose membrane (EMD Millipore, Billerica, MA, USA). The membrane was blocked by incubation with $5 \%(\mathrm{w} / \mathrm{v})$ nonfat milk in Tris-buffered saline with Tween-20 (Sigma-Aldrich; Merck Millipore). The membrane was then incubated with anit-IRAK1 (cat. no. sc-7883, 1:500, Santa Cruz Biotechnology, Inc., Dallas, TX, USA), anti-TRAF6 (cat. no. sc-7221, 1:500, Santa Cruz Biotechnology, Inc.), anti-HMGB-1 (cat. no. sc-135809, 1:500, Santa Cruz Biotechnology, Inc.), anti-TLR4 (cat. no. sc-293072, 1:500, Santa Cruz Biotechnology, Inc.), anti-MyD88 (cat. no. sc-11356, 1:500, Santa Cruz Biotechnology, Inc.), anti-NF-кB (cat. no. sc-7178, 1:500, Santa Cruz Biotechnology, Inc.) and anti-GAPDH (cat. no. AG019, 1:2,000, Beyotime Institute of Biotechnology) antibodies overnight at $4^{\circ} \mathrm{C}$. The blots were then incubated with anti-rabbit horseradish peroxidase-conjugated antibodies (cat. no. A0208, 1:5,000, Beyotime Institute of Biotechnology) for $1 \mathrm{~h}$ at room temperature. The protein blank was detected using enhanced chemiluminescence (Beyotime Institute of Biotechnology) method and images were captured using the Image Lab (version 3.0; Bio-Rad Laboratories, Inc., Hercules, CA, USA).

Statistical analysis. All data are expressed as the mean \pm standard deviation and analysed using SPSS software (version 19.0; IBM Corp., Armonk, NY, USA). One-way analysis of variance was performed to compare the differences between two groups, followed by Duncan's test. $\mathrm{P}<0.05$ was considered to indicate a statistically significant difference.

\section{Results}

Effect of dioscin on infarct volume and neurological scores in rats with ischemic stroke. In order to confirm the effect of dioscin on ischemic stroke, the infarct volume and neurological scores were determined. As shown in Fig. 2A, there were significant increases in infarct volume and neurological scores in the ischemic stroke group, compared with the sham control group. By contrast, treatment with dioscin significantly reduced the ischemic stroke-induced infarct volume and neurological scores in the ischemic stroke model (Fig. 2B).

Effect of dioscin on inflammatory responses in the rat ischemic stroke model. To further confirm the inhibitory effect of dioscin in the ischemic stroke rats, the activities of IL-le activities ofts, e respowere measured using ELISA kits.
As shown in Fig. 3A-D, the results indicated that there were increases in the activities of IL- $1 \beta$, IL- 6 and TNF- $\alpha$ and a decreased in the activity of IL-10 in the rats of the ischemic stroke model, compared with those in the control group. In addition, dioscin treatment significantly inhibited the activities of IL-1activities of igniin the rat ischemic stroke model (Fig. 3).

Effect of dioscin on activities of caspase-3/9 in the rat ischemic stroke model. To further confirm the involvement of caspase activation in the effect of dioscin on apoptosis in rats with ischemic stroke, caspase- 3 and caspase- 9 kits were used to measure these indices. The activities of caspase-3 and caspase- 9 in the rats of the ischemic stroke model were increased, compared with those in the sham control group (Fig. 4A and B). Treatment with dioscin significantly inhibited the activities of caspase-3 and caspase-9 activity in the ischemic stroke model (Fig. 4A and B).

Effect of dioscin on the activity of TGF- $\beta 1$ in the rat ischemic stroke model. To determine whether the effect of dioscin affects the activity of TGF-acin the rats in the ischemic stroke model, the activities of TGF-c swere measured using ELISA kits. Compared with that in the sham control group, the activity of TGF-actin the ischemic stroke group was enhanced (Fig. 5). Treatment with dioscin significantly suppressed the activity of TGF- $\beta 1$ in the ischemic stroke model (Fig. 5).

Effect of dioscin on the protein expression levels of IRAKI and TRAF6 in the rat ischemic stroke model. The present study examined the protein expression levels of IRAK1 and TRAF6 in the rats of the ischemic stroke model treated with dioscin. As shown in Fig. 6A-C, the protein expression levels of IRAK1 and TRAF6 in the ischemic stroke group were higher, compared with those of the sham control group. Dioscin markedly reduced the protein expression levels of IRAK1 and TRAF6 in the ischemic stroke model (Fig. 6A-C).

Effect of dioscin on the protein expression levels of HMGB-1 in the rat ischemic stroke model. The present study assessed whether dioscin affected the protein expression levels of HMGB-1 in the rats ischemic stroke model. As shown in Fig. 7A and B, the protein expression of HMGB-1 in the ischemic stroke group was increased, compared with that in the sham group. The induced protein expression of HMGB-1 
A
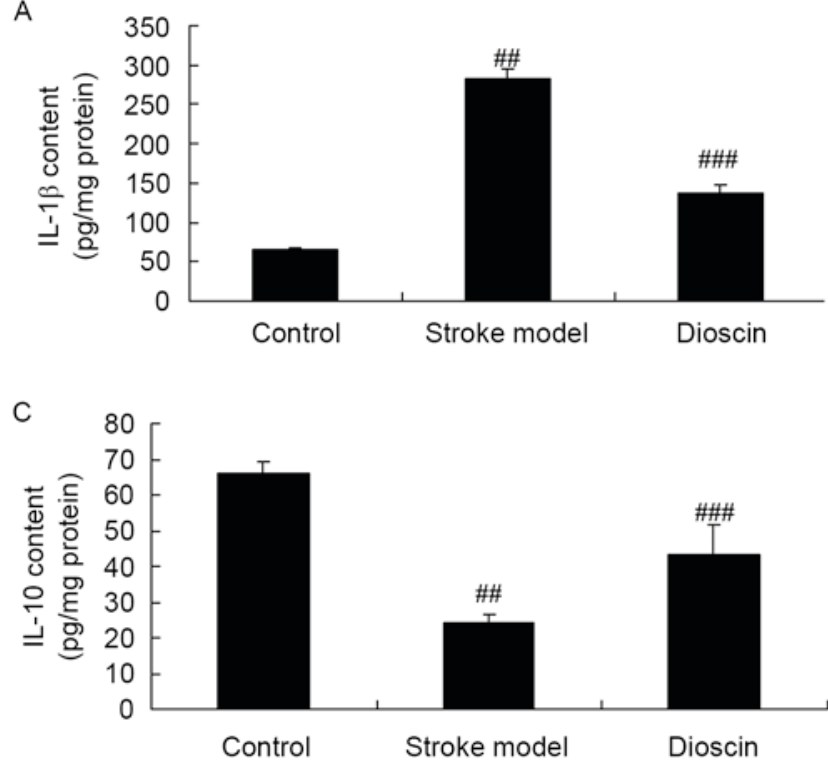

B
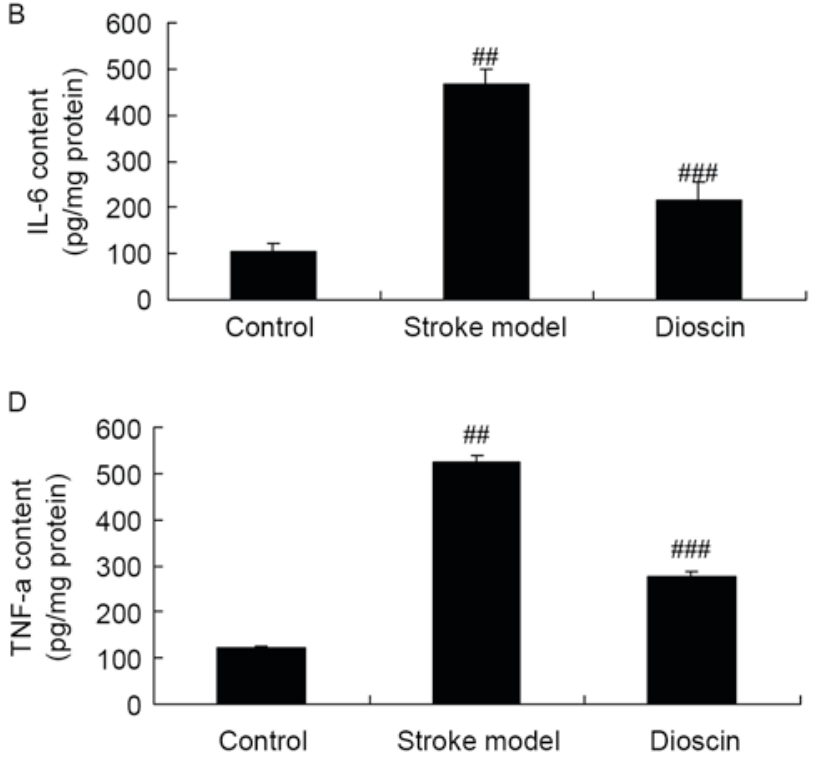

Figure 3. Effect of dioscin on inflammatory responses in ischemic stroke rats. Effects of dioscin on activities of (A) IL-1 1 , (B) IL-6, (C) IL-10 and (D) TNF-0 in ischemic stroke rats. Control, control group; Stroke, ischemic stroke model group; Dioscin, dioscin treatment group. ${ }^{\# \#} \mathrm{P}<0.01$, vs. control group; ${ }^{\# \# / 1} \mathrm{P}<0.01$, vs. ischemic stroke model group. IL, interleukin; TNF-group.ke, ischemic stroke m.
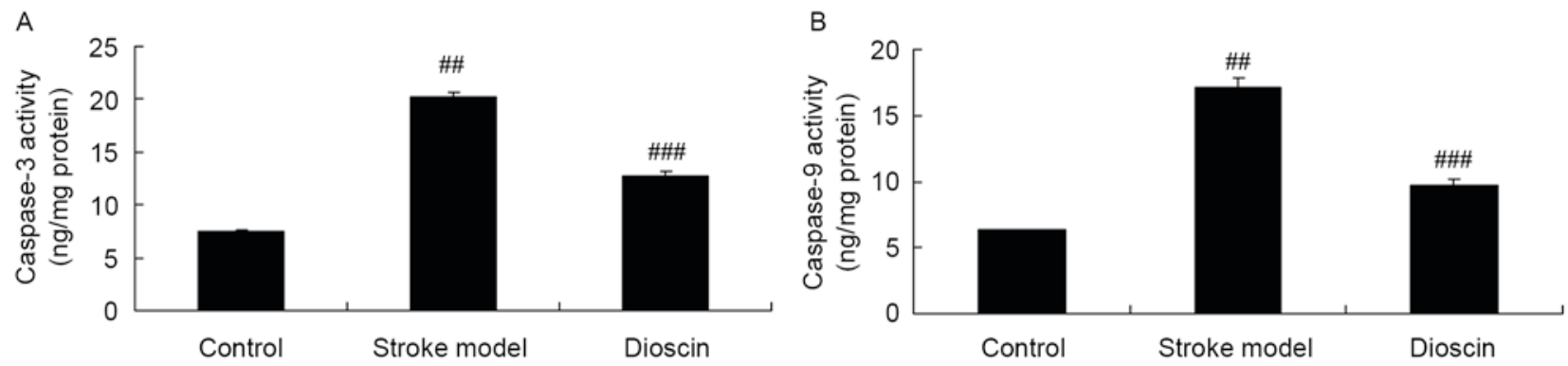

Figure 4. Effect of dioscin on activities of caspase-3/9 in ischemic stroke rats. Effects of dioscin on the activities of (A) caspase-3 and (B) caspase-9 in ischemic stroke rats. Control, control group; Stroke, ischemic stroke model group; Dioscin, dioscin treatment group. ${ }^{\# \#} \mathrm{P}<0.01$, vs. control group; ${ }^{\# \# "} \mathrm{P}<0.01$, vs. ischemic stroke model group.

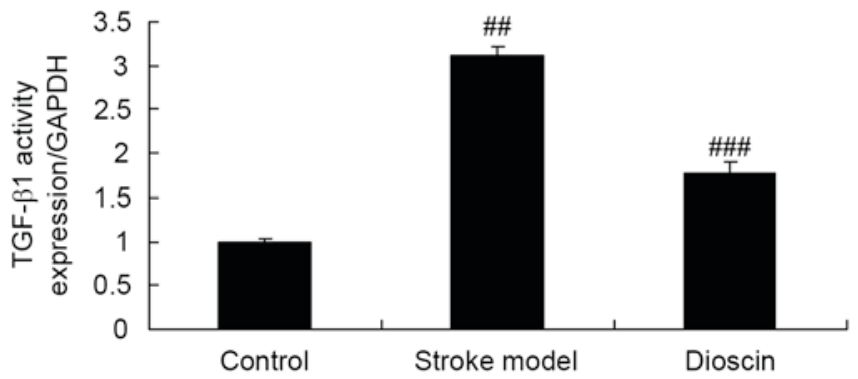

Figure 5. Effect of dioscin on the activity of TGF- $\beta 1$ in ischemic stroke rats. Effect of dioscin on the expression of TGF- $\beta 1$. Control, control group; Stroke, ischemic stroke model group; Dioscin, dioscin treatment group. ${ }^{\# \#} \mathrm{P}<0.01$, vs.

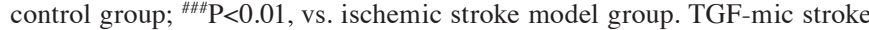
model growth factor- $\beta 1$.

in the ischemic stroke group was significantly suppressed by dioscin (Fig. 7A and B).

Effect of dioscin on the protein expression levels of TLR4 in the rat ischemic stroke model. In order to examine the effects of the administration of dioscin on stroke, western blot analysis was performed to measure the protein expression levels of TLR4 in the ischemic stroke group. As shown in Fig. 8A and $\mathrm{B}$, the protein expression level of TLR4 in the ischemic stroke group was markedly increased, compared with that on the sham control group. However, treatment with dioscin significantly suppressed the protein expression of TLR4 in the ischemic stroke group (Fig. 8A and B).

Effect of dioscin on the protein expression of MyD88 in the rats ischemic stroke model. The present study also examined the protein expression levels of MyD88 in rats with ischemic stroke treated with dioscin using western blotting analysis. As shown in Fig. 9A and B, the protein expression of MyD88 in ischemic stroke group was higher, compared with that in the sham control group. Dioscin treatment significantly suppressed the protein expression of MyD88 in the ischemic stroke group (Fig. 9A and B).

Effect of dioscin on the protein expression of $N F-\kappa B$ in the rat ischemic stroke model. To investigate the possible role 


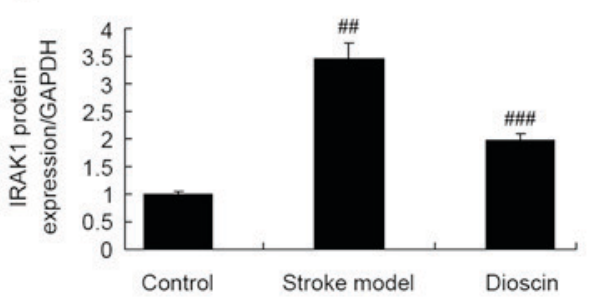

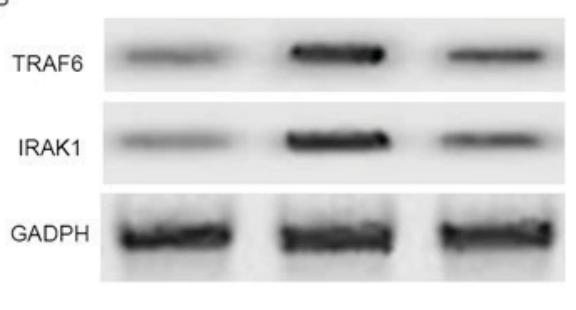

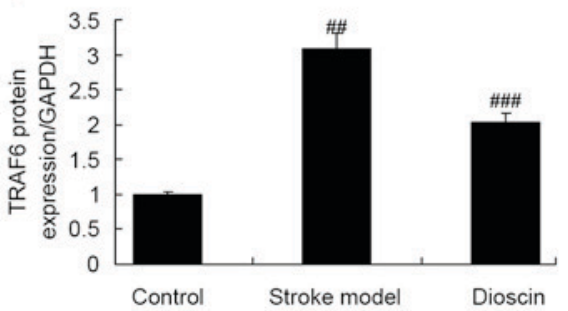

Figure 6. Effect of dioscin on protein expression levels of IRAK1 and TRAF6 in ischemic stroke rats. (B) Effects of dioscin on the protein expression levels of IRAK1 and TRAF6 were determined using western blot analysis. (A) Statistical analysis of the protein expression levels of IRAK1 and TRAF6 in ischemic stroke rats. Control, control group; Stroke, ischemic stroke model group; Dioscin, dioscin treatment group. ${ }^{\# /} \mathrm{P}<0.01$, vs. control group; ${ }^{\# \# /} \mathrm{P}<0.01$, vs. ischemic stroke model group. IRAK1, interleukin-1 receptor-associated kinase 1; TRAF6, tumor necrosis factor receptor-associated factor 6.

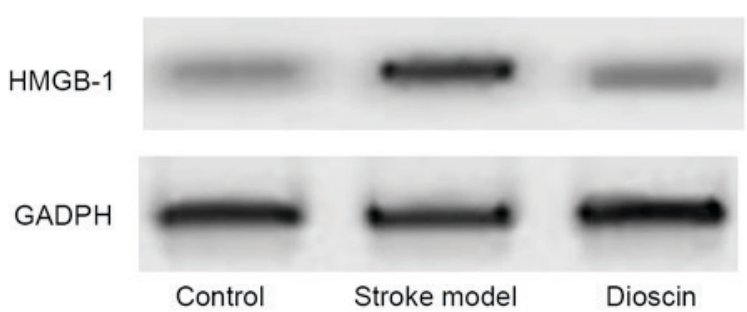

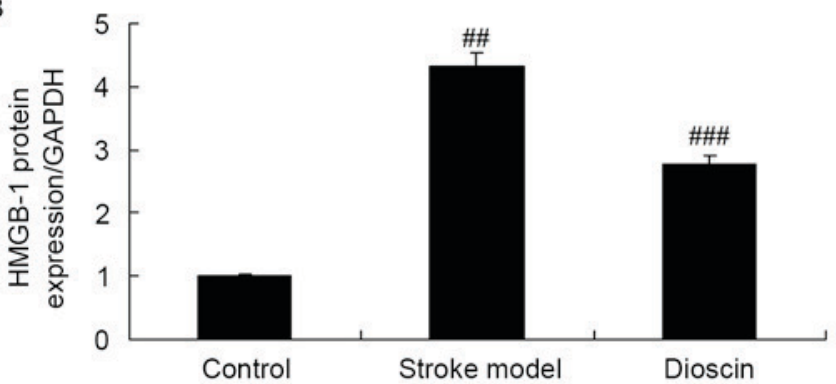

Figure 7. Effect of dioscin on protein expression levels of HMGB-1 in ischemic stroke rats. (A) Effect of dioscin on protein expression of HMGB-1 was determined using western blot analysis. (B) Statistical analysis of protein expression levels of HMGB-1 in ischemic stroke rats. Control, control group; Stroke, ischemic stroke model group; Dioscin, dioscin treatment group. ${ }^{\# \#} \mathrm{P}<0.01$, vs. control group; ${ }^{\# \# /} \mathrm{P}<0.01$, vs. ischemic stroke model group. HMGB-1, high-mobility group protein 1 .

A

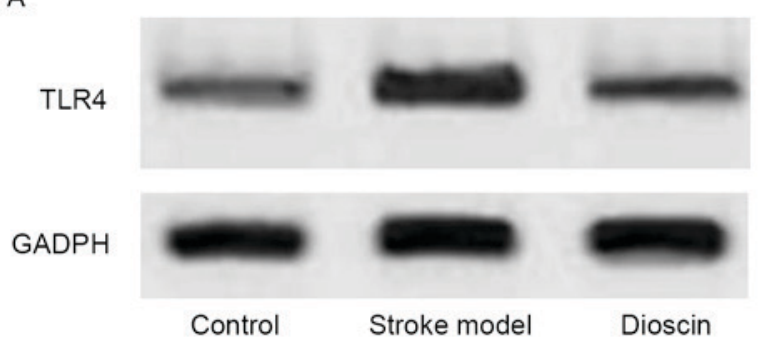

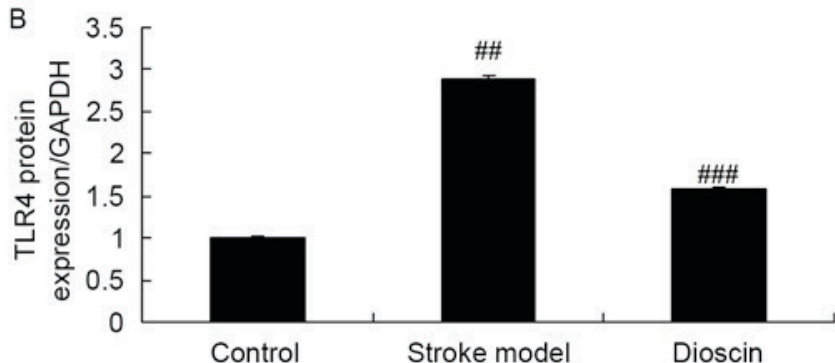

Figure 8. Effect of dioscin on protein expression levels of TLR4 in ischemic stroke rats. (A) Effect of dioscin on protein expression of TLR4 was determined using western blot analysis. (B) Statistical analysis of protein expression levels of TLR4 in ischemic stroke rats. Control, control group; Stroke, ischemic stroke model group; Dioscin, dioscin treatment group. ${ }^{\# \#} \mathrm{P}<0.01$, vs. control group; ${ }^{\# \# / ~} \mathrm{P}<0.01$, vs. ischemic stroke model group. TLR4, Toll-like receptor 4.

of $\mathrm{NF}-\kappa \mathrm{B}$ invpathways in the effects of dioscin on ischemic stroke, the protein expression levels of $\mathrm{NF}-\kappa \mathrm{B}$ were examined using western blot analysis. The results of the western blot analysis showed that the protein expression of $\mathrm{NF}-\kappa \mathrm{B}$ was significantly increased in the ischemic stroke group, compared with that in the sham control group. By contrast, dioscin treatment significantly suppressed the protein expression of $\mathrm{NF}-\kappa \mathrm{B}$ in the ischemic stroke group (Fig. 10A and B).

\section{Discussion}

Acute cerebral infarction is also known as apoplexy or cerebral apoplexy. It is one of the most complicated nervous system diseases with the highest level of damage. It has been ranked as the third leading cause of mortality and the leading contributor to disability (17). Following thrombosis, sudden interruption or cerebral blood flow or emboli, patients suffer from symptoms, including paralysis, language damage and eyesight loss (18). In addition, $<15 \%$ of cases are caused by hemorrhagic stroke or cardiac arrest. According to statistical data of the United States in 2014, the average rate of stroke occurrence was once every $40 \mathrm{sec}$, with associated mortality every $4 \mathrm{~min}$ (4). The estimated mortality rate has reached $41.6 \%$ and, with an increasingly aging population the rates are likely to increase correspondingly. The data obtained in the present study suggested that treatment with dioscin significantly reduced ischemic stroke-induced infarct volume and neurological scores in the ischemic stroke model.

The pathological and physiological mechanisms of cerebral ischemia/reperfusion injury include energy failure, excitability, amino acid toxicity, oxidative stress damage, nitric oxide, inflammatory reaction, cell apoptosis, mitochondria 
A

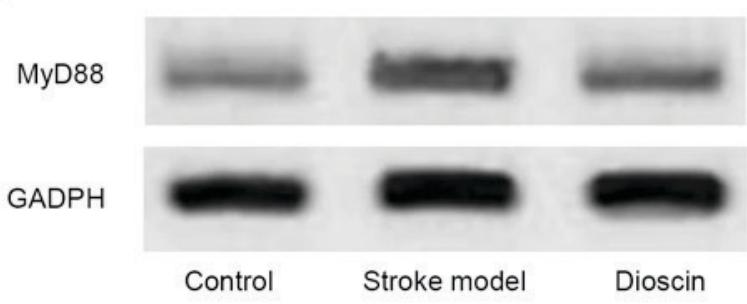

B

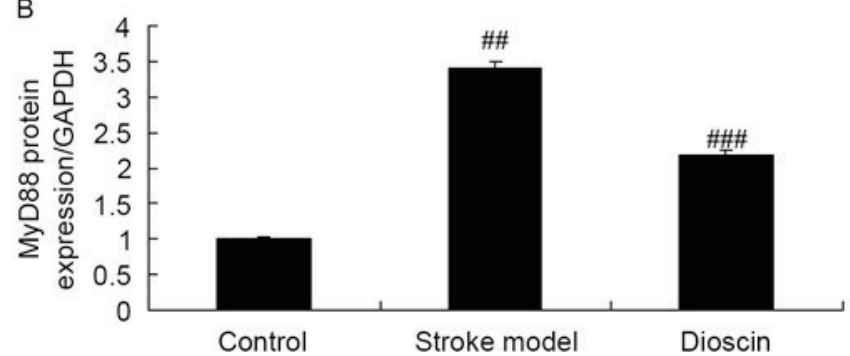

Figure 9. Effect of dioscin on protein expression levels of MyD88 in ischemic stroke rats. (A) Effect of dioscin on protein expression of MyD88 was determined using western blot analysis. (B) Statistical analysis of protein expression levels of MyD88 in ischemic stroke rats. Control, control group; Stroke, ischemic stroke model group; Dioscin, dioscin treatment group. ${ }^{\# \#} \mathrm{P}<0.01$, vs. control group; ${ }^{\# \# \#} \mathrm{P}<0.01$, vs. ischemic stroke model group. MyD88, myeloid differentiation factor 88 .

A

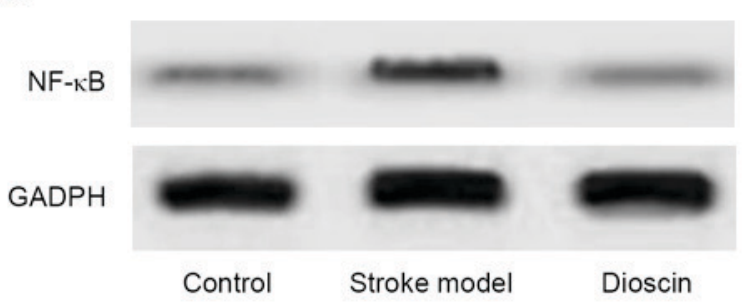

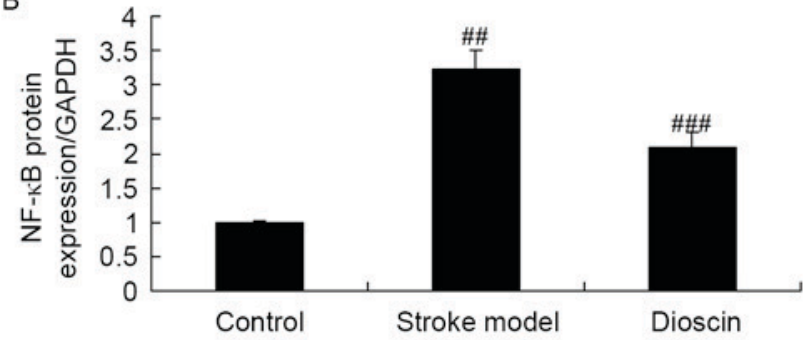

Figure 10. Effect of dioscin on protein expression levels of NF- $\kappa \mathrm{B}$ in ischemic stroke rats. (A) Effects of dioscin on protein expression of NF- $\kappa \mathrm{B}$ was determined using western blot analysis. (B) Statistical analysis of protein expression levels of NF-e in ischemic stroke rats. Control, control group; Stroke, ischemic stroke model group; Dioscin, dioscin treatment group. ${ }^{\# \#} \mathrm{P}<0.01$, vs. control group; ${ }^{\# \# \#} \mathrm{P}<0.01$, vs. ischemic stroke model group. NF-hem nuclear factor-r s.

stress and autophagy. On reviewing the time-scale of cerebral ischemia/reperfusion injury, inflammatory reactions become apparent up to $2 \mathrm{~h}$ following ischemia, and further aggravate cerebral tissue damage (19). As a result of hematoencephalic barrier effects, the inflammatory reactions following ischemia are divided into central sleep apnea and peripheral (20). Initially, at the early stage of cerebral ischemia/reperfusion injury, free radicals, cell toxicity and other substances released by the 'infarction core' activate the microglia inherent inflammatory cells in the central nervous system, and induce microglial cells to release TNF-roglia inherent inflammatory cells in the central nervous sfollowing ischemia (21). Tao et al confirmed that dioscin ameliorates inflammation through TLR4 signaling via the inhibition of HMGB-1 in an in vitro cerebral ischemia/reperfusion injury model (22).

These results showed that Dioscin treatment significantly inhibited the activities of IL- $1 \beta$, IL- 6 , TNF- $\alpha$, caspase- 3 and caspase- 9 in the ischemic stroke rats, which showed that the dioscin-induced inhibition of ischemic stroke was dependent on the inflammation and anti-apoptotic effects.

TLRs are one of the most important pattern recognition receptor families, and have key effects on the induction of inflammatory reactions and generation of the inflammatory medium process (23). All TLRs are enriched in the extracellular region of leucine-rich repeat sequences, and is necessary for recognizing pathogen-associated molecular patterns (PAMPs) (24). In addition, the intracellular Tol I-interleukin-1 receptor structural domain is required for initiating intracellular signal transduction. The aforementioned structure can recognize conserved molecular structures and microorganism products, including the endogenous molecular patterns of
PAMPs and tissue damage (25). A previous study have shown that the expression of TLR4 is upregulated in an ischemic stroke model (25), expressed predominantly on microglial cells and neurons. In addition, in a mouse model with TLR4 defect, nerve function loss and brain damage were less marked, compared with those in wild-type mice (26). TLR activation leads to the translocation of $\mathrm{NF}-\kappa \mathrm{B}$. It is a key regulatory factor mediating the expression of inflammatory mediators and the inflammatory reaction. Inhibiting its activity reduces brain damage (27). The results of the present study showed that dioscin significantly suppressed the activity of TGF- $\beta 1$, reduced the protein expression levels of IRAK1 and TRAF6, and suppressed the protein expression levels of HMGB-1 and TLR4 in the rats of the ischemic stroke model. Liu et al showed that dioscin alleviates alcoholic liver fibrosis through

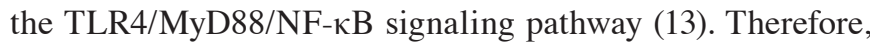
the effect of dioscin on ischemic stroke may be associated with the TLR4 pathway.

The TLR4/NF-kB signal transduction pathway activates downstream inflammatory cytokines, including TNF-a, IL-6, IL-10, C-X-C motif chemokine ligand-10, interferon-F and chemokines, and upregulates the expression of cell adhesion molecules. In addition, the expression of matrix metalloproteinase (MMP)-9 in ischemic brain tissue increases with pro-inflammatory effects $(11,28)$. The cytokines generated following ischemia can induce the generation of MMP, which causes an increase in vasopermeability and blood-brain barrier (BBB) damage. This further promotes microvascular basement membrane protein hydrolysis and vasogenic cerebral edema generation, and leads to increased cerebral ischemia, BBB destruction, encephaledema, neuronal cell death, and 
further aggravation of brain damage (10). Qi et al found that dioscin alleviates LPS-induced inflammatory kidney injury via the TLR4/MyD88 signaling pathway (12). The results of the present study showed that dioscin treatment significantly also suppressed the protein expression levels of MyD88 and NF-88 in the rats of the ischemic stroke model, and downregulated the TLR4/MyD88/NF-4/ pathway.

In conclusion, based on the observations of the present study, dioscin significantly reduced ischemic stroke-induced infarct volume and neurological scores, and inhibited ischemic stroke-induced inflammation and expression levels of TGF-ession levels ofced inflammain the ischemic stroke model. These findings provide novel insights into the mechanisms of dioscin as a potent anti-inflammatory agent, which may be used to treat ischemic stroke via the TLR4/MyD88/NF-reat ischemi Therefore, dioscin may have anti-inflammatory, anti-apoptotic and other effects, which require confirmation in the future, in addition to clinical application to provide further data to support the findings obtained in the present study.

\section{References}

1. Lin YN, Hu CJ, Chi JY, Lin LF, Yen TH, Lin YK and Liou TH: Effects of repetitive transcranial magnetic stimulation of the unaffected hemisphere leg motor area in patients with subacute stroke and substantial leg impairment: A pilot study. J Rehabil Med 47: 305-310, 2015.

2. Verdecchia P, Reboldi G, Angeli F, Trimarco B, Mancia G, Pogue J, Gao P, Sleight P, Teo K and Yusuf S: Systolic and diastolic blood pressure changes in relation with myocardial infarction and stroke in patients with coronary artery disease. Hypertension 65: 108-114, 2015.

3. Jusufovic M, Sandset EC, Bath PM, Karlson BW and Berge E; Scandinavian Candesartan Acute Stroke Trial Study Group: Effects of blood pressure lowering in patients with acute ischemic stroke and carotid artery stenosis. Int J Stroke 10: 354-359, 2015.

4. Muchada M, Rubiera M, Rodriguez-Luna D, Pagola J, Flores A, Kallas J, Sanjuan E, Meler P, Alvarez-Sabin J, Ribo M and Molina CA: Baseline National Institutes of Health stroke scale-adjusted time window for intravenous tissue-type plasminogen activator in acute ischemic stroke. Stroke 45: 1059-1063, 2014

5. Lindman BR, Zajarias A, Madrazo JA, Shah J, Gage BF, Novak E, Johnson SN, Chakinala MM, Hohn TA, Saghir M and Mann DL: Effects of phosphodiesterase type 5 inhibition on systemic and pulmonary hemodynamics and ventricular function in patients with severe symptomatic aortic stenosis. Circulation 125 : 2353-2362, 2012.

6. Xin Q, Cheng B, Pan Y, Liu H, Yang C, Chen J and Bai B: Neuroprotective effects of apelin-13 on experimental ischemic stroke through suppression of inflammation. Peptides 63: 55-62, 2015.

7. Boehme AK, McClure LA, Zhang Y, Luna JM, Del Brutto OH, Benavente OR and Elkind MS: Inflammatory markers and outcomes after lacunar stroke: Levels of inflammatory markers in treatment of stroke study. Stroke 47: 659-667, 2016.

8. Folyovich A, Biró E, Orbán C, Bajnok A, Varga V, Béres-Molnár AK, Vásárhelyi B and Toldi G: Relevance of novel inflammatory markers in stroke-induced immunosuppression. BMC Neurol 14: 41, 2014.

9. Zhang P, Guo ZF, Xu YM, Li YS and Song JG: N-Butylphthalide (NBP) ameliorated cerebral ischemia reperfusion-induced brain injury via HGF-regulated TLR4/NF- $\mathrm{B}$ signaling pathway. Biomed Pharmacother 83: 658-666, 2016.

10. Zhang B, Choi JJ, Eum SY, Daunert S and Toborek M: TLR4 signaling is involved in brain vascular toxicity of PCB153 bound to nanoparticles. PLoS One 8: e63159, 2013.

11. Ye L, Yang Y, Zhang X, Cai P, Li R, Chen D, Wei X, Zhang X, $\mathrm{Xu} \mathrm{H}, \mathrm{Xiao} J$, et al: The role of bFGF in the excessive activation of astrocytes is related to the inhibition of TLR4/NFKB signals. Int J Mol Sci 17: pii: E37, 2015.
12. Qi M, Yin L, Xu L, Tao X, Qi Y, Han X, Wang C, Xu Y, Sun H, Liu K and Peng J: Dioscin alleviates lipopolysaccharide-induced inflammatory kidney injury via the microRNA let-7i/TLR4/MyD88 signaling pathway. Pharmacol Res 111: 509-522, 2016.

13. Liu M, Xu Y, Han X, Yin L, Xu L, Qi Y, Zhao Y, Liu K and Peng J: Dioscin alleviates alcoholic liver fibrosis by attenuating hepatic stellate cell activation via the TLR4/MyD88/NF- $\kappa \mathrm{B}$ signaling pathway. Sci Rep 5: 18038, 2015.

14. Guo Y, Xing E, Song H, Feng G, Liang X, An G, Zhao X and Wang M: Therapeutic effect of dioscin on collagen-induced arthritis through reduction of Th1/Th2. Int Immunopharmacol 39: 79-83, 2016.

15. Lee HJ, Park JS, Yoon YP, Shin YJ, Lee SK, Kim YS, Hong JH, Son KH and Lee CJ: Dioscin and methylprotodioscin isolated from the root of Asparagus cochinchinensis suppressed the gene expression and production of airway MUC5AC mucin induced by phorbol ester and growth factor. Phytomedicine 22: 568-572, 2015.

16. Zhao X, Xu L, Zheng L, Yin L, Qi Y, Han X, Xu Y and Peng J: Potent effects of dioscin against gastric cancer in vitro and in vivo. Phytomedicine 23: 274-282, 2016.

17. Cramer SC and Hill MD; REGENESIS-LED Investigators: Human choriogonadotropin and epoetin alfa in acute ischemic stroke patients (REGENESIS-LED trial). Int J Stroke 9: 321-327, 2014.

18. Punt M, van Alphen B, van de Port IG, van Dieën JH, Michael K, Outermans $\mathrm{J}$ and Wittink $\mathrm{H}$ : Clinimetric properties of a novel feedback device for assessing gait parameters in stroke survivors. J Neuroeng Rehabil 11: 30, 2014.

19. Wang LC, Wu CL, Cheng YY and Tsai KJ: Deletion of nuclear localizing signal attenuates proinflammatory activity of prothymosin-alpha and enhances its neuroprotective effect on transient ischemic stroke. Mol Neurobiol 54: 582-593, 2017.

20. Liu W, Wang X, Zheng Y, Shang G, Huang J, Tao J and Chen L: Electroacupuncture inhibits inflammatory injury by targeting the miR-9-mediated NF- $\kappa$ B signaling pathway following ischemic stroke. Mol Med Rep 13: 1618-1626, 2016.

21. Liesz A and Kleinschnitz C: Editorial: Mechanisms of neuroinflammation and inflammatory neurodegeneration in acute brain injury. Front Cell Neurosci 9: 300, 2015.

22. Tao X, Sun X, Yin L, Han X, Xu L, Qi Y, Xu Y, Li H, Lin Y, Liu K and Peng J: Dioscin ameliorates cerebral ischemia/reperfusion injury through the downregulation of TLR4 signaling via HMGB-1 inhibition. Free Radic Biol Med 84: 103-115, 2015.

23. Vartanian KB, Stevens SL, Marsh BJ, Williams-Karnesky R, Lessov NS and Stenzel-Poore MP: LPS preconditioning redirects TLR signaling following stroke: TRIF-IRF3 plays a seminal role in mediating tolerance to ischemic injury. J Neuroinflammation 8: $140,2011$.

24. Derkow K, Krüger C, Dembny P and Lehnardt S: Microglia induce neurotoxic IL-17+ $\gamma \delta$ T cells dependent on TLR2, TLR4, and TLR9 activation. PLoS One 10: e0135898, 2015.

25. Belinga VF, Wu GJ, Yan FL and Limbenga EA: Splenectomy following MCAO inhibits the TLR4-NF- $\mathrm{KB}$ signaling pathway and protects the brain from neurodegeneration in rats. J Neuroimmunol 293: 105-113, 2016.

26. Sun M, Deng B, Zhao X, Gao C, Yang L, Zhao H, Yu D, Zhang F, $\mathrm{Xu} \mathrm{L}$, Chen $\mathrm{L}$ and Sun X: Isoflurane preconditioning provides neuroprotection against stroke by regulating the expression of the TLR4 signalling pathway to alleviate microglial activation. Sci Rep 5: 11445, 2015.

27. Zhang J, Wu Y, Weng Z, Zhou T, Feng T and Lin Y: Glycyrrhizin protects brain against ischemia-reperfusion injury in mice through HMGB1-TLR4-IL-17A signaling pathway. Brain Res 1582: 176-186, 2014.

28. Zhang J, Fu B, Zhang X, Chen L, Zhang L, Zhao X, Bai X, Zhu C, Cui L and Wang L: Neuroprotective effect of bicyclol in rat ischemic stroke: Down-regulates TLR4, TLR9, TRAF6, NF-кB, MMP-9 and up-regulates claudin-5 expression. Brain Res 1528: 80-88, 2013. 